\title{
Estimation of the blood velocity spectrum using a recursive lattice filter
}

\author{
Jensen, Jørgen Arendt; Buelund, Claus; Jørgensen, Allan; Munk, Peter
}

Published in:

Proceedings of the 1996 IEEE Ultrasonic Symposium

Link to article, DOI:

10.1109/ULTSYM.1996.584210

Publication date:

1996

Document Version

Publisher's PDF, also known as Version of record

Link back to DTU Orbit

Citation (APA):

Jensen, J. A., Buelund, C., Jørgensen, A., \& Munk, P. (1996). Estimation of the blood velocity spectrum using a recursive lattice filter. In Proceedings of the 1996 IEEE Ultrasonic Symposium (Vol. 1-2, pp. 1221-1224). I E E E International Ultrasonics Symposium. Proceedings https://doi.org/10.1109/ULTSYM.1996.584210

\section{General rights}

Copyright and moral rights for the publications made accessible in the public portal are retained by the authors and/or other copyright owners and it is a condition of accessing publications that users recognise and abide by the legal requirements associated with these rights.

- Users may download and print one copy of any publication from the public portal for the purpose of private study or research.

- You may not further distribute the material or use it for any profit-making activity or commercial gain

- You may freely distribute the URL identifying the publication in the public portal 


\title{
ESTIMATION OF THE BLOOD VELOCITY SPECTRUM USING A RECURSIVE LATTICE FILTER
}

\author{
Jørgen Arendt Jensen, Claus Buelund, Allan Jørgensen and Peter Munk \\ Department of Information Technology, Build. 344, \\ Technical University of Denmark, DK-2800 Lyngby, Denmark
}

\section{Abstract}

In medical ultrasound the blood velocity distribution in a vessel can be found by emitting a pulsed field into the patient. The field is then scattered by the tissues and the red blood cells, and a single complex sample set is acquired at the depth of interest for each pulse emitted. This gives a sampled signal in the audio range due to the slow movement of the scatterers through the measurement gate. The velocity distribution within the gate can then be determined by Fourier transforming the sampled signal. The signals acquired for showing the blood velocity distribution are inherently non-stationary, due to the pulsatility of the flow. All current signal processing schemes assume that the signal is stationary within the window of analysis, although this is an approximation.

In this paper a recursive least-squares lattice filter is used for finding a parametric model for the velocity distribution. A new set of complex coefficients is calculated for each point in time, and it is thus possible to track the non-stationary properties of the stochastic velocity signal. The dynamic characteristics of the non-stationarity are incorporated through an exponential decay factor, that sets the exponential horizon of the filter. A factor close to 1 gives a long horizon with low variance estimates, but can not track a highly non-stationary flow. Setting the factor is therefore a compromise between estimate variance and the filter's dynamic adaptation. Using a lattice filter gives a structure that is easy and robust, when implemented with fixed point arithmetic.

The procedure has been tested on both simulated and invivo data, and gives spectral estimates quite different from the normal FFT approach. Synthetic data were generated based on the measured time evolution of the spatial mean velocity in the femoral artery. The smooth theoretical velocity distribution is then known and can be compared to the estimated distribution. Using 8 parameters a very smooth estimate of the velocity distribution is seen, more in line with the actual distributions that always will be smooth. Setting the exponential decay factor to 0.99 gives satisfactory results for in-vivo data from the carotid artery. The filter can easily be implemented using a standard fixed-point signal processing chip for real-time processing.

\section{Introduction}

The distribution of blood velocities in human vessels can be measured non-invasively by using ultrasound. The measurement is performed by emitting a number of consecutive ultrasound pulses. A single complex sample set is acquired at the depth of interest for each pulse emitted. This gives a sampled signal in the audio range due to the slow movement of the scatterers through the measurement gate [1]. The frequency of the received digital signal is given by:

$$
f_{r}=f_{e} \frac{2 v_{z}}{c}
$$

where $f_{e}$ is the emitted frequency, $c$ is the speed of sound, and $v_{z}$ is the blood velocity along the ultrasound direction of propagation. The received frequency, $f_{r}$, is thus proportional to the blood velocity. In general, a velocity distribution is found in the vessel giving rise to a distribution of frequen. cies. The received signal's spectrum corresponds to this distribution. An example is shown in Fig. 1. Here the ideal velocity distribution as a function of time is shown for a femoral artery. The distribution was calculated from the Fourier components for the mean velocity in a vessel as given by Evans et al. [2], and using the theory developed by Womersley [3] and Evans [4]. The distribution is continuous, as the velocity is a continuous function of space and time.

The distribution can be estimated from the acquired digital ultrasound signal. This is done by short time Fourier transformation, where the signal is divided into small overlapping segments and Fourier transformed. The resulting spectra are displayed side by side in a sonogram display as shown in Fig. 2. The displayed spectra are stochastic, due to the underlying stochastic signal, and a typical speckle appearance of the sonogram is seen. The sonogram is thus not a faithful display of the velocity distribution, and it would be appropriate to find a method for displaying the continuous and smooth distribution. 


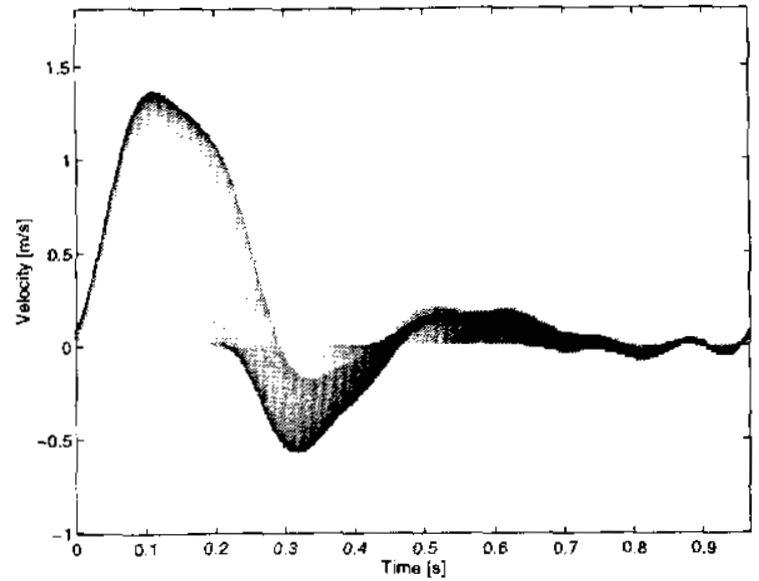

Figure 1: Velocity distribution in the femoral artery.

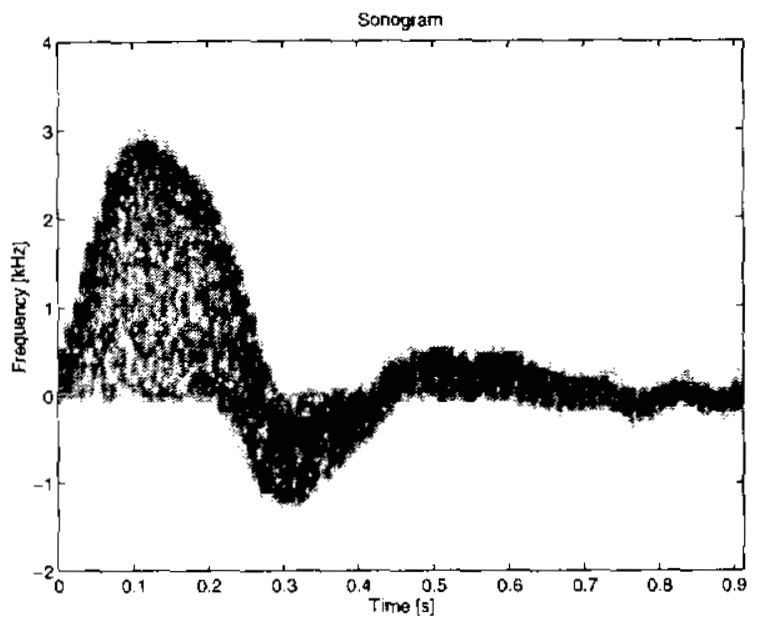

Figure 2: Sonogram of velocity distribution in the femoral artery.

\section{Parametric spectral estimation}

One method for finding a continuous spectrum from a stochastic signal is to introduce a parametric model. The model then fits the power density spectrum of the process, and the stochastic nature of the signal is modeled by the stochastic input signal driving the model. The most common models are the AR (AutoRegressive) model and the ARMA (AutoRegressive Moving Average) model. The ARMA model can be difficult to use, due to the indirect estimation of the MA part. The AR model will be used in this work, due to the fast and numerically stable algorithms for estimating it. The AR model is given by:

$y(n)=-a_{1} y(n-1)-a_{2} y(n-2) \cdots-a_{M} y(n-M)+e(n)$ where $y(n)$ is the observed signal $e(n)$ is the white, stochastic signal driving the model, and $M$ is the model order. The model is estimated by minimizing the variance of the prediction error:

$$
V=E\left\{(y(n)-\hat{y}(n \mid \theta))^{2}\right\}
$$

where $y(n \mid \theta)$ is the prediction of $y(n)$ and $\theta$ contains the estimated parameters. Some authors have found the AR coefficients using the Yule-Walker equations directly by dividing the data into segments, and then determined the parameters [5], [6]. This, however, assumes that the signal is stationary within the segment, which in general is not a valid assumption. There is therefore a compromise between selecting a small segment, where the signal is quasi-stationary, and at the same time getting enough data for a low-variance estimate. The situation can be improved by using a timerecursive estimation scheme, where the parameters for the process are continuously estimated as a function of time. The non-stationarity is then handled by introducing an exponential decay $\lambda$, in which the lag to the current estimation time determines the weight of the data. The exponential factor is adjusted to the rate of change in spectral properties of the signal. The time horizon in number of samples is given by

$$
N=\frac{1}{1-\lambda}
$$

\section{Recursive least squares lattice filter}

A number of different methods for implementing a recursive least squares estimate of the AR parameters exits. The recursive least squares lattice filter as developed by Morf et al. (1977) offers a number of advantages. It can be used on non-stationary signals, uses few calculations, and converges rapidly. Further, the lattice structure is numerically stable and is suited for fixed-point implementation.

The least squares lattice filter can be written as [8]:

TIME RECURSION:

$$
\begin{aligned}
& \text { for } n=1 \text { to } N \\
& \begin{array}{ll}
f_{0}(n) & =b_{0}(n)=y(n) \\
F_{0}(n) & =B_{0}(n)=\lambda F_{0}(n-1)+|y(n)|^{2} \\
\gamma_{0}(n-1) & =1 \\
a_{0}(n) & =1 \\
c_{0}(n-1) & =1
\end{array}
\end{aligned}
$$

\section{ORDER RECURSION:}

$$
\begin{aligned}
& \text { for } m=1 \text { to } M \\
& \qquad \begin{aligned}
\Delta_{m-1}(n)= & \lambda \Delta_{m-1}(n-1) \\
& +\frac{b_{m-1}(n-1) f_{m-1}^{*}(n)}{\gamma_{m-1}(n-1)}
\end{aligned}
\end{aligned}
$$




$$
\begin{aligned}
\Gamma_{f, m}(n)= & -\frac{\Delta_{m-1}(n)}{B_{m-1}(n-1)} \\
\Gamma_{b, m}(n)= & -\frac{\Delta_{m-1}^{*}(n)}{F_{m-1}(n-1)} \\
f_{m}(n)= & f_{m-1}(n)+\Gamma_{f, m}^{*}(n) b_{m-1}(n-1) \\
b_{m}(n)= & b_{m-1}(n-1)+\Gamma_{b, m}^{*}(n) f_{m-1}(n) \\
F_{m}(n)= & F_{m-1}(n)-\frac{\left|\Delta_{m-1}(n)\right|^{2}}{B_{m-1}(n-1)} \\
B_{m}(n)= & B_{m-1}(n-1)-\frac{\left|\Delta_{m-1}(n)\right|^{2}}{F_{m-1}(n)} \\
\gamma_{m}(n-1)= & \gamma_{m-1}(n-1)-\frac{\left.b_{m-1}(n-1)\right|^{2}}{B_{m-1}(n-1)} \\
a_{m}(n)= & {\left[\begin{array}{c}
a_{m-1}(n) \\
0
\end{array}\right]+} \\
& \Gamma_{f, m}(n)\left[\begin{array}{c}
0 \\
c_{m-1}(n-1)
\end{array}\right] \\
c_{m}(n)= & {\left[\begin{array}{c}
0 \\
c_{m-1}(n-1)
\end{array}\right]+} \\
& \Gamma_{b, m}(n)\left[\begin{array}{c}
a_{m-1}(n) \\
0
\end{array}\right]
\end{aligned}
$$

$x^{*}$ denotes the complex conjugate. The variables are:

$$
\begin{array}{ccl}
y & - & \text { Input signal } \\
f_{m} & - & \text { Forward a posteriori prediction error } \\
b_{m} & - & \text { Backward a posteriori prediction error } \\
\Gamma_{f} & - \text { Forward reflection coefficient } \\
\Gamma_{b} & - \text { Backward reflection coefficient } \\
\lambda & - & \text { Exponential decay factor } \\
a & - & \text { AR parameters }
\end{array}
$$

Division by a very small number can take place during the initial phase of the filtration. A divisor below $10^{-6}$ is thus discarded, and the term with the division is neglected. The filter is initialized by setting:

$$
\begin{aligned}
& \Delta_{m-1}(0)=0 \\
& F_{m-1}(0)=\delta \\
& B_{m-1}(0)=\delta
\end{aligned}
$$

where $\delta$ is some small number ( 0.01 here). The AR filter coefficients are then contained in the matrix $a$, and can be used for finding the parametric estimate of the power spectrum by:

$$
P(f, n)=\frac{\sigma_{e}^{2}}{\left|1+\sum_{i=1}^{M} a_{i}(n) \exp (-j 2 \pi f i \Delta T)\right|^{2}}
$$

where $\sigma_{e}^{2}$ is the variance of $e(n)$. This need not be determined since $\sigma_{e}^{2}$ is essentially constant over the heart cycle. Note that the filter estimates complex AR parameters, and the sign of the velocities can be displayed. The parametric sonogram is then found by continuously estimating the AR parameters and at regular intervals evaluating (5) and displaying the result.

All coefficients in the lattice filter are normalized to be between -1 and +1 , which makes it very easy to scale the filter for fixed-point implementation. The number of calculations

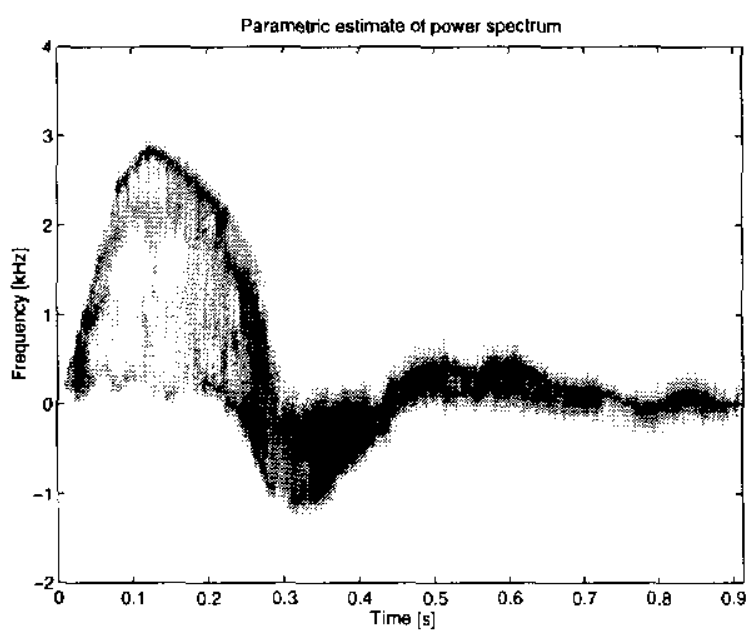

Figure 3: Parameteric sonogram from artificial data from the femoral artery. An 8th order AR model is used with $\lambda=0.99$.

to be performed per sample is proportional to $M$ and is on the order of $60 \mathrm{M}$ multiplications and additions and $16 \mathrm{M}$ divisions per sample, well within the reach of modern signal processing chips.

\section{Examples}

The first example is run on data from simulated flow in the femoral artery. The data was generated by the method described in Section 1. The lattice filter algorithm was run using the complex data and a filter order of 8 . The exponential decay factor was set to 0.99 . The resulting parametric sonogram is shown in Fig. 3. This should be compared to the sonogram for the same situation shown in Fig. 2. A smooth velocity distribution is now seen, which better resembles the actual continuous distribution from Fig. 1. It is thus possible to estimate the continuous distribution, when the underlying data is noise free.

The second example is for in-vivo data from the carotid artery of a healthy 29 -years old male. A B\&K Medical 3535 ultrasound scanner with a $5 \mathrm{MHz}$ type 8545 convex array transducer was used for the acquisition. The scanner was connected to our dedicated sampling system [9], which used a sampling frequency of $15 \mathrm{MHz}$, and acquired $50 \mathrm{RF}$ samples for each pulse emission. The pulse repetition frequency was $5 \mathrm{kHz}$, and a total of 19,000 lines or 3.8 seconds of data was acquired.

The RF data was preprocessed by the matched filter/Hilbert transform approach described by Jensen (1996). A filter matched to the emitted pulse is first applied to the data, and then a Hilbert transform is used to calculate the imaginary part of the signal. One complex set of samples is then ex- 


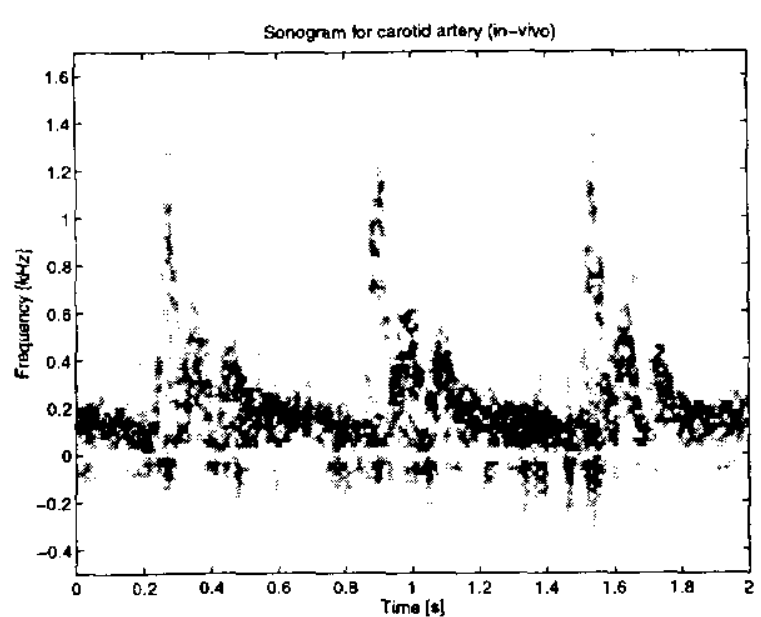

Figure 4: Sonogram from the carotid artery.

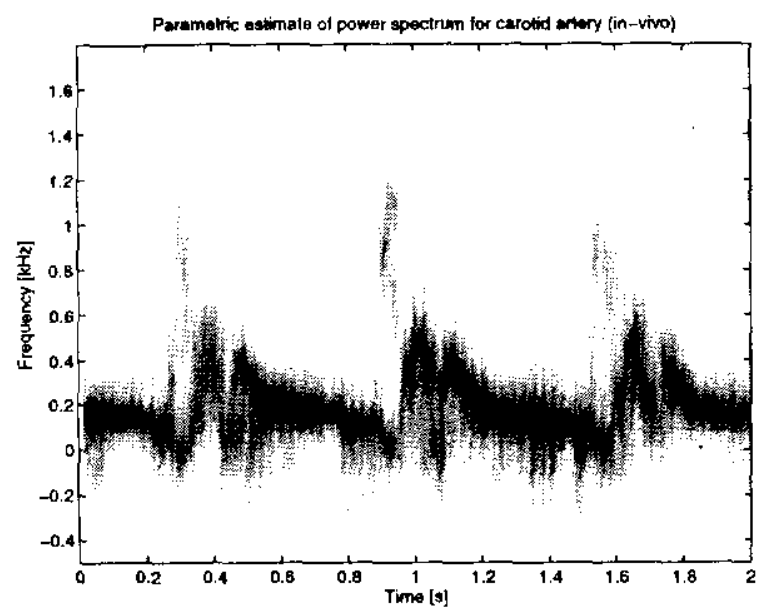

Figure 5: Parametric sonogram from the carotid artery.

tracted for each line, and this complex signal is used for spectral estimation and AR estimation.

The sonogram is shown in Fig. 4. A typical pulsatile waveform is seen along with the normal speckle pattern in the sonogram. Note also that the signal-to-noise ratio is quite low. The parametric sonogram is shown in Fig. 5 for a model order of 8 and $\lambda=0.99$. A much smoother appearance is seen than for the sonogram, more in keeping with the underlying velocity distribution. The estimates are more noisy than for the synthetic signals, That is due to the high noise level, where essentially white noise is present in the signal. This disturbs the estimation process, since the filter seeks to model this broad-band noise.

\section{Conclusion}

It has been shown that parametric spectral estimation using a recursive lattice filter can yield smooth velocity distribution estimates that are closer to the actual underlying distributions. A very good resemblance to the theoretical distribution was attained for synthetic data from the femoral artery. The invivo data was less conclusive, since the signal-to-noise ratio for these data was quite low. A study using higher quality data is, thus, needed.

\section{References}

[1] J. A. Jensen. Estimation of Blood Velocities Using Ultrasound: A Signal Processing Approach. Cambridge University Press, New York, 1996.

[2] D. H. Evans, W. N. McDicken, R. Skidmore, and J. P. Woodcock. Doppler Ultrasound, Physics, Instrumentation, and Clinical Applications. John Wiley \& Sons, New York, 1989.

[3] J. R. Womersley. Oscillatory motion of a viscous liquid in a thin-walled elastic tube. I: The linear approximation for long waves. Phil. Mag., 46:199-221, 1955.

[4] D. H. Evans. Some aspects of the relationship between instantaneous volumetric blood flow and continuous wave Doppler ultrasound recordings III. Ultrasound Med. Biol., 9:617-623, 1982.

[5] R. 1. Kitney and D. P. Giddens. Analysis of blood velocity waveforms by phase shift averaging and autoregressive spectral estimation. J. Biomech. Eng., 105:398-401, 1983.

[6] P. J. Vaitkus and R. S. C. Cobbold. A comparative study and assessment of Doppler ultrasound spectral estimation techniques, Part I: Estimation methods. Ultrasound Med. Biol., 14:661-672, 1988.

[7] M. Morf, A. Vieira, and D. T. Lee. Ladder forms for identification and speech processing. In Proc. 1977 IEEE Conf. Decision and Control, pages 1074-1078, New Orleans, 1977.

[8] S. Haykin. Adaptive Filter Theory. Prentice-Hall, Englewood Cliffs, New Jersey, 1986.

[9] J. A. Jensen and J. Mathorne. Sampling system for in vivo ultrasound images. In Med. Imag. V Symp., volume SPIE Vol. 1444, pages 221-231, 1991. 